\title{
Simulations of spatially and angle-resolved vibrational electron energy loss spectroscopy for a system with a planar defect
}

\author{
Paul M. Zeiger $\circledast^{*}$ and Ján Rusz $\odot$ \\ Department of Physics and Astronomy, Uppsala University, P.O. Box 516, 75120 Uppsala, Sweden
}

(Received 29 April 2021; revised 3 August 2021; accepted 12 August 2021; published 10 September 2021)

\begin{abstract}
Recent developments in experiments with vibrational electron energy loss spectroscopy (EELS) have revealed spectral shape variations at spatial resolutions down to the atomic scale. Interpretation in terms of local phonon density of states enables their qualitative understanding, yet a more detailed analysis is calling for advances in theoretical methods. Recently, we have presented a frequency resolved frozen phonon multislice method for simulations of vibrational EELS [P. M. Zeiger and J. Rusz, Phys. Rev. Lett. 124, 025501 (2020).]. Detailed simulations for a plane-wave electron beam scattering on vibrating hexagonal boron nitride are presented in a companion paper [P. M. Zeiger and J. Rusz, Phys. Rev. B 104, 104301 (2021)]. Here we present simulations of vibrational EELS assuming a convergent electron probe of nanometer size and atomic size on a hexagonal boron nitride structure model with a planar defect. With a nanometer beam we observe spectral shape modifications in the presence of the defect, which are correlated with local changes of the phonon density of states. With an atomic-sized electron beam, we observe the same, although with better contrast. In addition, we observe atomic-level contrast and atomic scale spectral shape modifications, which are particularly strong for small detector collection angles.
\end{abstract}

DOI: 10.1103/PhysRevB.104.094103

\section{INTRODUCTION}

Heat management is a major design limitation of integrated circuits today [1,2] and Moore's law is at the same time reaching its limits [3]. Advances in the field of phononic and thermoelectric materials allow for the control of phonons over large frequency regions and make it thereby possible to control the flow of heat [4]. Understanding the flow of heat at the nanometer and subnanometer levels might lead to further progress in these fields and a technique which can deliver this spatial resolution is a prerequisite for such developments.

(Scanning) transmission electron microscopy [(S)TEM] routinely allows to reach subangstrom spatial resolution [5-10] and thanks to recent advances in monochromators it offers an energy resolution of electron energy loss spectroscopy (EELS) down to $4.2 \mathrm{meV}[11,12]$. Unprecedented experiments such as mapping of bulk and surface modes of nanocubes [13], investigations of the nature of polariton modes in van der Waals crystals [14], temperature measurement at the nanoscale $[15,16]$, identification and mapping of isotopically labeled molecules [17], positionand momentum-resolved mapping of phonon modes [18-20], atomic resolution phonon spectroscopy [21,22], functional

*paul.zeiger@physics.uu.se

Published by the American Physical Society under the terms of the Creative Commons Attribution 4.0 International license. Further distribution of this work must maintain attribution to the author(s) and the published article's title, journal citation, and DOI. Funded by Bibsam. group mapping [23], single stacking fault [24], and singleatom vibrational spectroscopy [25] were enabled by these advances in STEM instrumentation.

Venkatraman et al. have observed atomic scale spectral changes in a silicon ( $\mathrm{Si}$ ) crystal, attributing them to selective phonon excitations as a function of electron beam impact parameter [22]. In another work, Hage et al. have shown, on a monolayer of graphene containing a single Si impurity atom, that vibrational STEM-EELS spectra show atomic scale changes near the Si impurity [25]. Both works recognized the necessity of a general simulation method to address the complexity of observed phenomena, such that would be capable to treat arbitrary three-dimensional structures, including eventual defects, and allow for general beam and detector geometry. Recently, Yan et al. have presented space- and angle-resolved measurements of vibrational EELS modifications in the presence of a stacking fault in silicon carbide, which were correlated with the local phonon density of states; however, the effects of electron beam propagation and scattering have not been considered [24].

A theoretical analysis of phonon scattering in TEM and its prospects for high-resolution imaging was discussed by Rez well before the advent of the above-mentioned instrumental advances [26]. Estimations of particular phonon scattering cross sections have been reported in Ref. [27], and recently phonon EELS was treated with a Bloch-waves-based theory in Ref. [28]. Forbes et al. have introduced an approach to phonon scattering based on Born-Oppenheimer theory, today referred to as the quantum excitation of phonons (QEP) model [29]. Using this approach simulations have shown that atomic scale contrast can be observed using phonon scattering intensities [30], though it should be mentioned that even if the QEP 
model allows to isolate the scattering intensity due to phonon processes, this approach does not provide spectroscopic information. Forbes and Allen have presented a simulation of phonon EELS spectra of silicon for a plane-wave incoming beam [31]. Dwyer reported inelastic multislice simulations adapted to phonon excitations, which led to a prediction of high-resolution phonon EELS [32], later realized in experiment [33]. The prospects of spatial resolution with vibrational EELS and the impact of dipole scattering were analyzed in Ref. [34]. Recently, Konečná et al. have reported a theory of phonon mapping and isotope identification [35].

All the above-mentioned spectroscopic simulation methods require an explicit knowledge of phonon modes, which can become very costly for large structure models, for example those containing interfaces or defects. Recently we have reported on a frequency-resolved frozen phonon multislice (FRFPMS) method allowing efficient simulations of vibrational spectra, fully considering dynamical diffraction effects as well as beam and detector setups [36]. In Ref. [37] we analyze the method in more detail and present results of calculations for a plane-wave electron beam. Importantly, the FRFPMS method scales linearly with the number of atoms, making it particularly suitable for simulations of phonon EELS in large structure models with tens or even hundreds of thousands of atoms, such as heterostructures or structures with defects.

Here we present detailed simulations of spatially and angle-resolved vibrational EELS on a model system of hexagonal boron nitride (h-BN) with $\mathrm{AA}^{\prime}$ stacking. Two structure models are being considered. The first one is a periodic crystal without any structural defects [37]. The second model contains a planar defect [an antiphase boundary (APB)] and the defect plane is oriented parallel to the electron beam. While such a system is qualitatively similar to a stacking fault studied by Yan et al. [24], we note that here every atomic column consists of the same number of B and N elements and, as such, it is a priori not likely to be revealed by high-angle annular dark field (HAADF) imaging. We show that the FRFPMS method resolves both angle-resolved spectral shape variations on a nanometer scale as well as atomic scale spectral changes analogous to those reported in previous experimental works. Using a large off-axis detector geometry offers a clear detection of the defect, both with nanometer-sized probe and atomic-sized probe. The latter reveals minor atomic scale spectral shape variations. A nanometer-sized electron probe gives furthermore access to the local phonon band structure using either small collection angles or $(q, E)$ mapping $[37,38]$.

\section{METHODS}

Most of the computational methods and parameters used here follow the choices made in the companion paper, where we describe the FRFPMS method in detail and consider the case of parallel illumination [37]. For completeness, we briefly summarize the methods and parameter settings here, highlighting the differences in methodology and parameters between both works.

We have performed independent simulations of an APBfree bulk h-BN structure model and of a structure model containing two grains of bulk h-BN and an APB at both
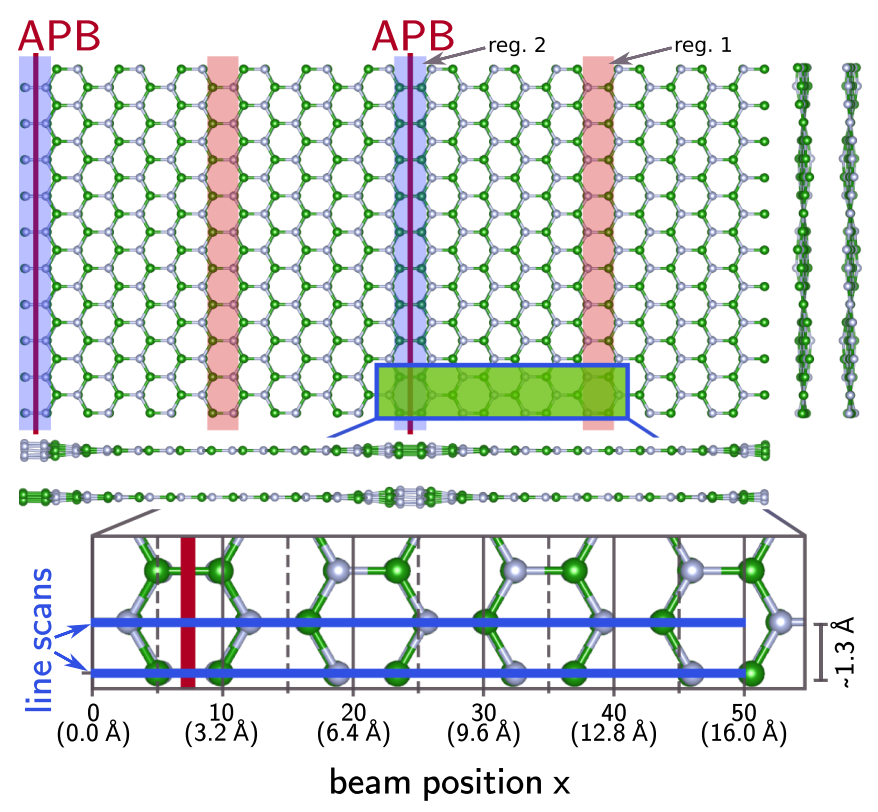

FIG. 1. Relaxed h-BN structure with an antiphase boundary. The supercell viewed along the $z$ direction is displayed together with two side views of the two upper layers. Two types of areas are highlighted in red (region 1) and blue (region 2), referring to an "in-grain" region and APB region, respectively. The areas scanned with atomic-sized electron beam (green rectangle) and line scans (thick blue lines) used in the subsequent analysis are highlighted in the bottom panel.

interfaces between the grains (we apply periodic boundary conditions). Both structure models consist of the same total number of atoms $\left(N_{\mathrm{at}}=22080\right)$ and h-BN layers $\left(N_{\text {lay }}=46\right)$ in $\mathrm{AA}^{\prime}$ stacking order. Our APB model can be obtained by slicing the pristine h-BN structure once perpendicular to the h-BN layers (here the $z$ direction) and shifting then one of the two obtained "halves" by one interlayer distance along the $z$ direction. As a result, the APB plane consists solely of B-B and $\mathrm{N}-\mathrm{N}$ bonds. Electronic structure calculations show that such an APB is metastable in monolayer h-BN $[39,40]$. The relaxed orthogonal simulation boxes have sizes of $52.01 \AA \times$ $25.02 \AA \times 149.71 \AA$ and $52.08 \AA \times 25.13 \AA \times 148.65 \AA$ for models without and with APB, respectively, and periodic boundary conditions were enforced in all three spatial directions. The structurally relaxed APB model, highlighting the atomic displacements in the neighborhood of the APB in the side views, is visualized in Fig. 1.

Structural relaxation as well as molecular dynamics calculations were performed with the LAMMPS software [41]. We have employed a so-called extended Tersoff potential for boron nitride (BN-ExTeP) developed by Los et al. [42] for the description of intralayer interactions. Contrary to the widely used Tersoff potential for boron nitride [43], this potential contains a description of boron-boron and nitrogen-nitrogen bonds, which is essential for our system. The BN-ExTeP has been successfully applied to defects in monolayer h-BN before [42]. For the description of interlayer interactions we have used a potential of Ouyang et al., which was specifically optimized for the description of bulk h-BN [44]. In addition, a shielded Coulomb potential accounts for partial charges on atoms between different layers [45]. 
Phonon calculations (not shown), which were performed using a combination of the PHONOLAMMPS [46] and PHONOPY software packages $[47,48]$, showed no imaginary frequencies for the structurally relaxed APB model. Furthermore, classical molecular dynamics simulations at constant pressure of $0.0 \mathrm{bar}$ and for temperatures of up to $1200 \mathrm{~K}$ showed no signs of structural reconfiguration of the APB, confirming the stability of the structure. That being said, such an APB has not yet been reported in h-BN to the best of our knowledge. Here it fulfills predominantly the role of a model system, but also of a potentially interesting prediction that could be probed by vibrational EELS experiments.

Nonequilibrium molecular dynamics calculations using the generalized Langevin equation have been done within LAMMPS using a hot-spot thermostat $[49,50]$ with a width of $\Delta \omega=2.5 \mathrm{THz}$. Energy bins range from $11.5 \mathrm{THz}$ up to $51.5 \mathrm{THz}$ with a step of $2.5 \mathrm{THz}$. In relation to Ref. [37], we use here the coarser of the two energy grids treated there. Nevertheless, it was shown that the effective energy resolution is in both cases close to $10 \mathrm{meV}$. Considering that in STEM calculations we need to analyze significantly more electron beam positions, this choice is computationally more efficient. The base temperature $T_{\text {base }}$ for the white-noise thermostat was set to zero and its damping parameter to $1 / \gamma_{\text {base }}=0.1 \mathrm{ps}$. The temperature at the peak of the hot-spot thermostat was set to $T_{\max }=300 \mathrm{~K}$ and the hot-spot damping parameter to $1 / \gamma=0.5 \mathrm{ps}$. The time step in these simulations was set to $0.5 \mathrm{fs}$.

In total $n_{\text {snap }}=225$ snapshots were generated per energy bin by the nonequilibrium molecular dynamics simulations, starting after 25 ps of equilibration, taking a snapshot every 1 ps until the total trajectory length of $0.25 \mathrm{~ns}$. The lateral multislice grid consisted of $1008 \times 480$ points. We have used the automatic potential slicing option of DRPROBE [51].

In all visualizations of vibrational EELS below we show spectra multiplied by the energy loss. This is motivated by observations made in Ref. [37] as well as by the first Born approximation expression for the inelastic scattering cross section containing the factor $1 / \omega_{\mathbf{q}, n}$ in all transition matrix elements, where the $\mathbf{q}, n$ indexes a specific phonon mode. Energy-multiplied spectra are visually more easily comparable to the (local) phonon density of states, which aids the discussion.

HAADF $Z$-contrast simulations were performed using the standard frozen phonon multislice method [52] by averaging over 60 snapshots. In contrast to the FRFPMS method, these snapshots were generated using constant-temperature and constant-volume molecular dynamics simulations at $300 \mathrm{~K}$ using a combination of microcanonical ensemble with Langevin dynamics, but otherwise using the same structure model and multislice parameters as for the FRFPMS simulations.

\section{RESULTS}

\section{A. Local phonon density of states}

Figure 2(a) shows a comparison of local phonon density of states (LPDOS) obtained for a system with and without defect. In Fig. 1 we have highlighted two areas for evaluation of the LPDOS. What is marked as "reg. 1" is a set of
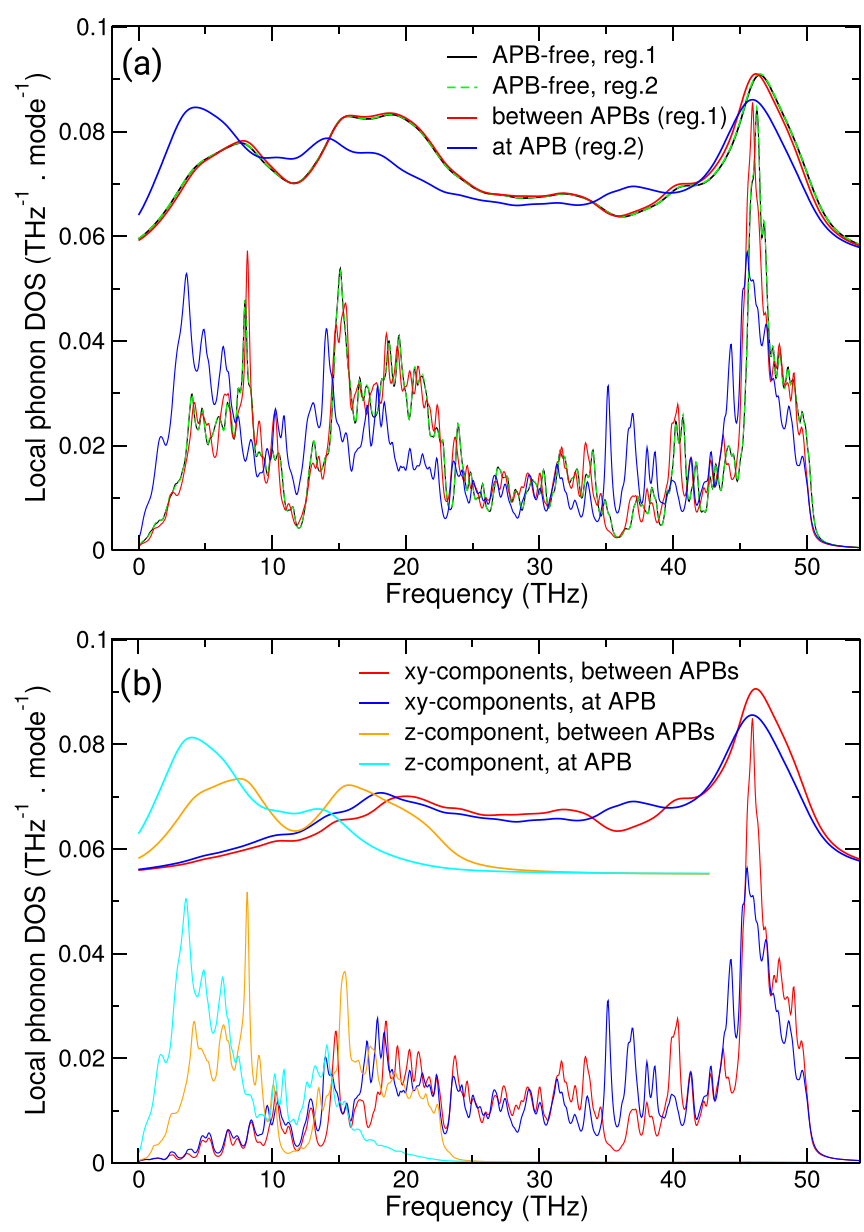

FIG. 2. (a) Comparison of local phonon densities of states calculated on a model with and without an antiphase boundary, respectively. (b) Decomposition of the local phonon densities of states calculated for the model with antiphase boundary into their $z$ components (out of plane) and $x y$ components (in plane). In both panels, two local regions have been probed (see Fig. 1). Lorentzian broadening of two different full widths at half maximum were used, namely, 0.3 and $3.0 \mathrm{THz}$. The more broadened curves are vertically offset by $0.055 \mathrm{THz}^{-1}$ mode $^{-1}$.

atomic columns forming hexagons, that are positioned as far as possible from the two APBs. The label "reg. 2" then marks hexagons containing atoms forming the APB. For consistence and comparison purposes, we have defined the same areas within the APB-free structure model.

When using the structure model without defect, the LPDOS from both areas is expected to be the same and this is indeed the case (see the overlapping black and dashed green curves). The LPDOS is dominated by an optical peak at a frequency of around $46 \mathrm{THz}$ and a set of less pronounced peaks at lower frequencies.

For a sufficiently large structure model, the LPDOS from region 1 in the APB structure model should match the LPDOS of the defect-free structure model. As Fig. 2(a) shows, the corresponding LPDOS (red curve) follows the LPDOS of the APB-free model very closely and we observe only a small $(\sim 0.2 \mathrm{THz})$ redshift of the optical peak and a similar blueshift of acoustic modes. The broadened LPDOS of both 

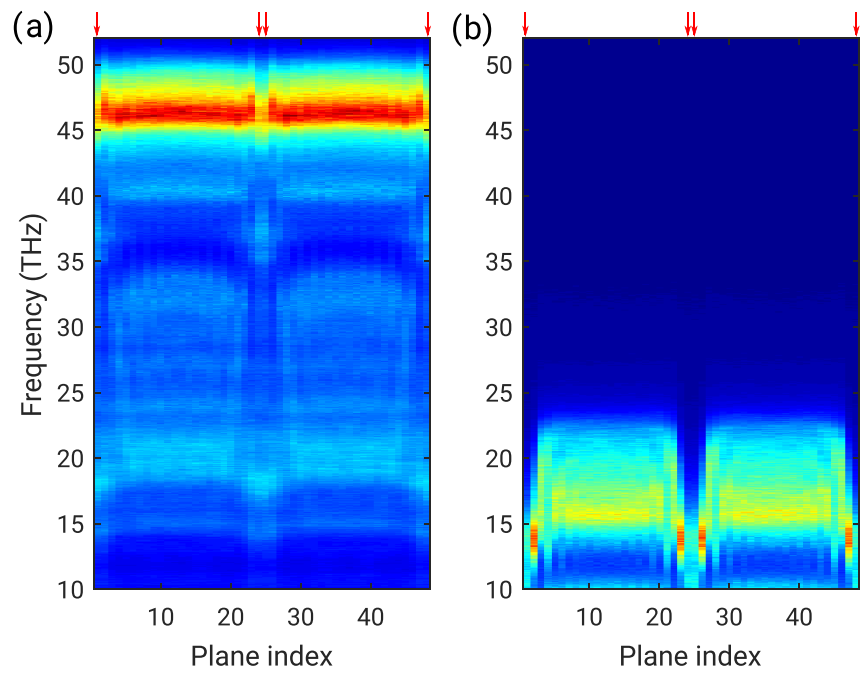

FIG. 3. Projected LPDOS grouped according to the 48 atomic planes along the $x$ direction of our h-BN model with APB (see Fig. 1). The horizontal axis labels atomic plane indices from 1 to 48. Atomic planes 1, 24, 25, and 48, marked by small red arrows, are directly involved in the APB. (a) An in-plane component of the LPDOS and (b) a $z$ component, respectively.

structure models are practically indistinguishable. Overall the agreement of the LPDOS of APB and defect-free structure is excellent, suggesting that the structure model containing a defect is large enough to encompass regions where the impact of defects on the LPDOS is negligible.

As expected, the LPDOS of the defect region (blue curve) differs significantly from the other three LPDOS. It has enhanced acoustic modes at low frequencies and the optical peak has a deformed shape, with a maximum redshifted by approximately $0.6 \mathrm{THz}$ compared to the defect-free regions. Several other differences can be observed, for example, an appearance of vibrational modes within the energy range 35$39 \mathrm{THz}$, where the defect region LPDOS is much higher than in the defect-free region. Another pronounced difference is a reduction of the LPDOS in the defect region around $20 \mathrm{THz}$.

Figure 2(b) shows a decomposition of the LPDOS calculated for the structure model with defect into its components due to vibrations in the in-plane $(x y)$ and out-of-plane $(z)$ directions. Since the electron beam propagates along the $z$ direction, it is mostly sensitive to in-plane atomic vibrations [53]. This will be important when the calculated vibrational EELS spectra will be correlated with the LPDOS. For instance, from approximately 15 to $23 \mathrm{THz}$ the total LPDOS is significantly larger in the defect-free region; however, the decomposition into in-plane and out-of-plane components reveals that this is almost entirely due to vibrations in the $z$ direction, which are expected to influence the electron beam only negligibly. The in-plane LPDOS in this energy region is of comparable amplitude in both regions, with the defect region LPDOS being on average somewhat higher.

The degree of locality of changes in the LPDOS induced by the APB can be qualitatively deduced from Fig. 3. There we plot the LPDOS for the 48 atomic planes of our simulation box along the $x$ direction as a heat map. The LPDOS of atoms within APB planes are distinctly different from the LPDOS of

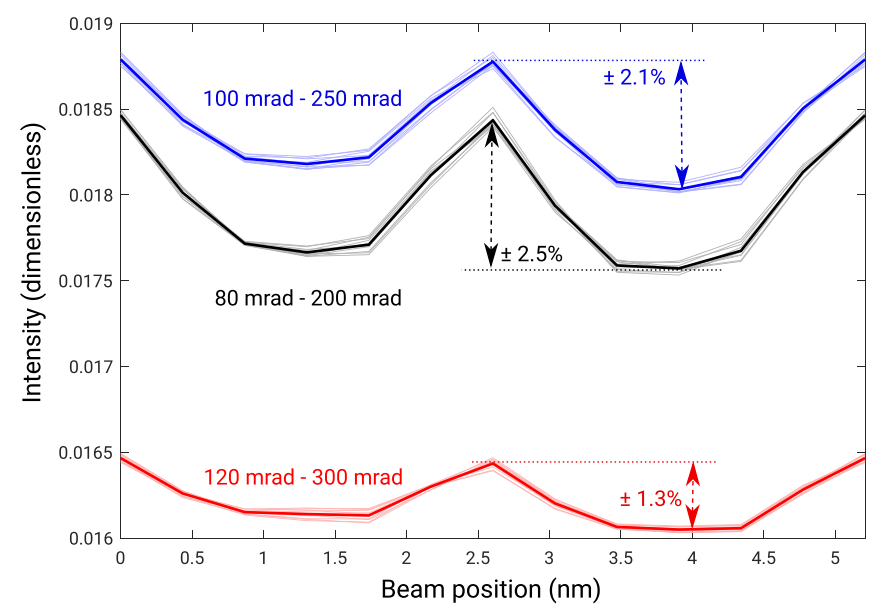

FIG. 4. High-angle annular dark field profiles across the whole structure model with two defect planes. Ten individual profiles for different beam $y$ coordinates are plotted with thin lines of brighter colors. Their average is shown with thicker lines of saturated colors. Three different settings of inner and outer collection angles are indicated. The relative variation of intensity is given as the ratio of difference and sum of the maximal and the minimal intensity of each averaged profile.

atoms far from the APB throughout the whole energy range. This suggests that not only a few modes are influenced by the defect, but rather many modes are impacted throughout the entire range of vibrational frequencies. The total LPDOS is mostly constant within planes 3-22 and 27-46, showing a strong localization of the modification of vibrations due to APB. On the other hand, there are energy ranges where the influence of the defect on the vibrations is less localized. Note particularly the arcs between 30 and $34 \mathrm{THz}$ and defect modes around 18 and $37 \mathrm{THz}$ in the in-plane component, Fig. 3(a), or out-of-plane vibrations of frequencies between 10 and 15 THz, Fig. 3(b).

\section{B. Vibrational EELS at nanometer scale}

We start our analysis with a nanometer-sized electron beam, to check whether the above-mentioned LPDOS differences can be detected in simulated vibrational EELS spectra. We have set up a calculation with a convergent probe of 3 mrad semiangle, which leads to a beam full width at half maximum of approximately $0.9 \mathrm{~nm}$ at an acceleration voltage of $60 \mathrm{kV}$ (neglecting any source-size broadening or aberrations). We have evaluated a grid of $12 \times 10$ beam positions, evenly spanning the whole structure model. That corresponds to a STEM sampling with steps of $0.43 \mathrm{~nm}$ along the $x$ direction and $0.25 \mathrm{~nm}$ along the $y$ direction, respectively.

Figure 4 shows calculated HAADF profiles across the structure model with defect planes. By symmetry, we would not expect strong differences for beam positions differing by $y$ coordinate only, because the beam is too large for any atomicscale contrast and the local differences in atomic positions and bonds are only along the $z$ direction-referring to the small displacement of atoms near the planar defect, seen in $x z$ or $y z$ projections of Fig. 1. This is indeed the case, as can be seen in Fig. 4. Profiles shown with thick lines are averages over 
ten beam positions along the $y$ axis. The figure also includes the ten individual linear profiles with thin lines of a brighter color and they are obviously very close to each other and thus also to their average. Three different settings of inner and outer collection angles have been considered. Each of the profiles displays local maxima at the positions of defect planes. The HAADF intensity variation is, however, relatively weak. Defining it as a ratio of the maximal minus the minimal profile intensity and of their sum, the intensity variation ranges between $1.3 \%$ and $2.5 \%$; i.e., it is a relatively weak effect. Yet it is nonzero, despite the same elemental composition of every single atomic column within the structure model. In the following paragraphs we will address the question whether vibrational EELS has the capability to highlight the presence of the defect planes.

First we will analyze spectra calculated with a large offaxis detector. Specifically, we have set the detector center to (60 mrad,0) and its radius (collection semiangle) to $25 \mathrm{mrad}$. In Fig. 5(a) we show ten spectra from the defect region (blue curves) and ten spectra from the defect-free region (red curves). Within each region, the ten individual spectra match closely each other, similarly as the individual HAADF profiles in Fig. 4. Mutual differences of individual spectra provide to a certain degree an estimation of the statistical error in our sampling. However, it should be pointed out that the ten individual spectra are correlated to some degree, because the same structure snapshots have been used in their calculations and thus the same combinations of excited phonons are present. From a different perspective, atomic displacements in a given sample region do not unambiguously determine the displacements elsewhere in the sample, despite the reduced portfolio of available phonon modes within an energy bin; therefore, the results suggest that the averaging over 225 snapshots provides a well-converged result-at least, for the selected large offaxis detector.

The difference of the spectra from the two regions is significantly stronger than the spread of the spectra within each region. This provides a theoretical support for the experimental observation that a nanometer-sized electron beam is indeed sufficiently small to distinguish a planar defect [24].

The spectral differences in Fig. 5(a) match the qualitative differences between the LPDOS curves in Fig. 2 well, such as the slight redshift of the optical phonon peak (barely seen at the energy resolution of $2.5 \mathrm{THz}$, which corresponds to approximately $10 \mathrm{meV}$ ), enhanced acoustic region (here specifically the region just above $10 \mathrm{THz}$ ), or the more intense defect-region spectrum between 35 and $40 \mathrm{THz}$, when compared to the spectrum from the defect-free region. The stronger spectrum from the defect-free region around $30 \mathrm{THz}$ comes likely from a similar feature in the LPDOS just above $30 \mathrm{THz}$. In relative terms, the modest enhancement of the acoustical modes within the defect region is more in line with the in-plane LPDOS component than with the total LPDOS (see Fig. 2), as was anticipated. It is somewhat surprising that the intensity and shape of optical peaks come out rather similar in both spectra-this is likely a consequence of relatively low energy resolution and $2.5 \mathrm{THz}$ distance between energy bins.

A step further in mapping the nanometer-scale spectral shape variations has been inspired by experiments of Yan et al.
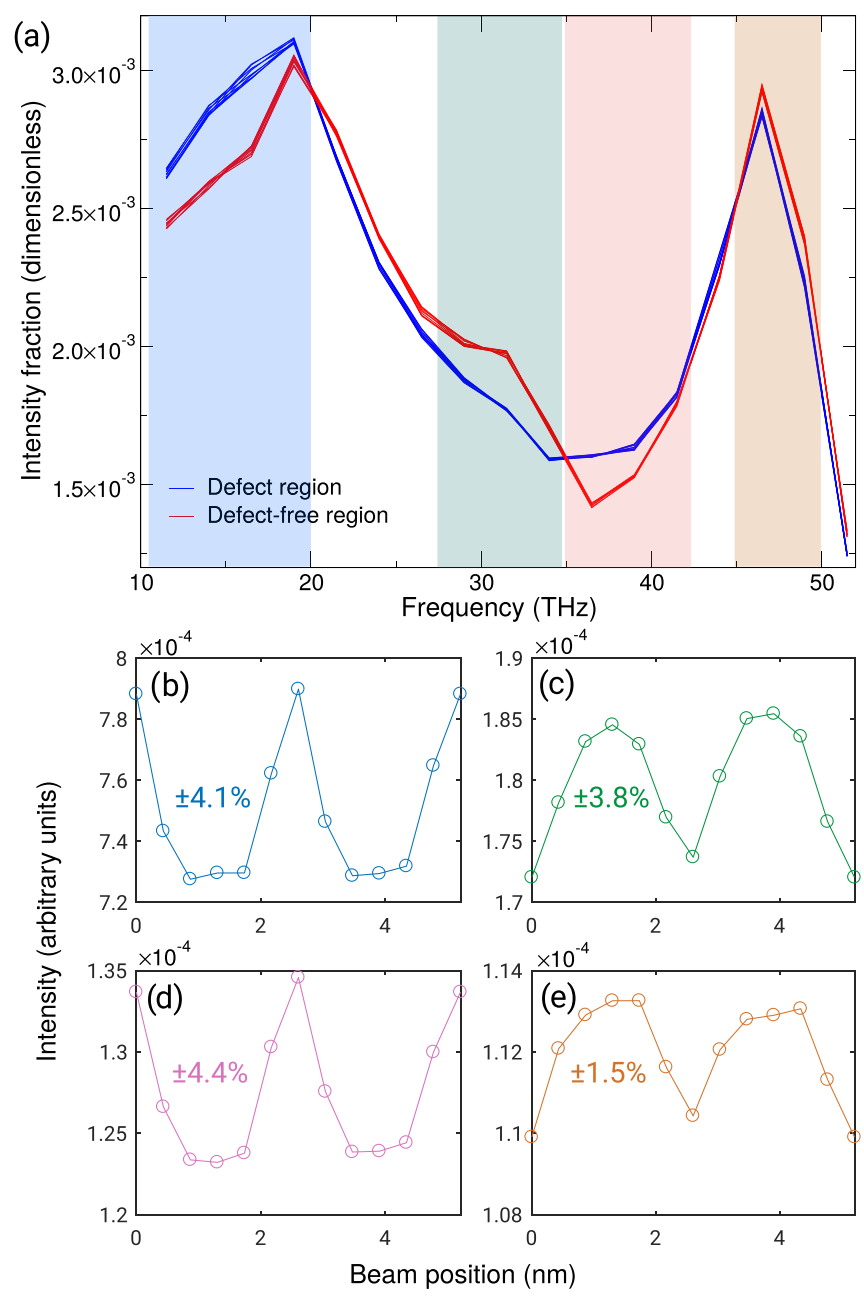

FIG. 5. (a) Vibrational EELS spectra calculated within the two regions defined in Fig. 1 assuming a nanometer-sized electron beam with convergence semiangle of $3 \mathrm{mrad}$. Within each of the regions ten spectra were evaluated at different beam $y$ coordinates. Four different frequency regions are marked by shading. (b)-(e) Linear profiles across the whole structure model containing two planar defects are shown with frequency ranges and color coding corresponding to the shaded regions in (a). Beam position step is approximately $0.43 \mathrm{~nm}$ resulting in 12 evenly spaced beam positions. Percentages refer to the intensity variations, defined as in Fig. 4.

[24]. As mentioned above, we have run spectral simulations for a grid of $12 \times 10$ beam positions evenly covering the whole structure model. The 12 evenly spaced beam positions correspond to a step of approximately $0.43 \mathrm{~nm}$ in the direction perpendicular to the defect planes. Encouraged by very small spectral differences observed along the $y$ direction in Fig. 5(a), the ten evenly spaced beam positions in the $y$ direction have been averaged to improve statistics. In Fig. 5(a) we have highlighted four different energy ranges of interest, over which we have integrated the vertically averaged spectra and plotted them as intensity profiles across the structure model in the $x$ direction. One can clearly observe the variation of the intensities correlating with the positions of the two planar defects in all highlighted energy ranges, an observation analogous to the enhancement of the acoustic modes in the vicinity of a 
stacking fault in SiC observed in Fig. 3 of Ref. [24]. Note also that in comparison with the HAADF signal, the percentage of the intensity variation across the defect is increased in the vibrational spectra, ranging from $\pm 1.5 \%$ for optical modes up to more than $\pm 4 \%$ for both acoustic modes. We note here that the larger delocalization of the spectral modifications due to the defect in the frequency range $30-34 \mathrm{THz}$ is well reflected in Fig. 5(c), where we do not observe a flat plateau inside APB-free regions, contrary to other panels of Fig. 4 corresponding to energy ranges, where the defect modes do not extend that far away from APB.

In order to get a deeper insight into the vibrational scattering of a nanometer-sized electron beam, we explore the information contained in spectra simulated with a smallcollection-angle detector. Specifically, we set the detector collection semiangle to $3 \mathrm{mrad}$ and we scan its center along specific lines within the diffraction plane. We focus on the region of scattering angles between 30 and $110 \mathrm{mrad}$, where the inelastic (phonon) scattering represents a sizable fraction of the total scattering cross section.

Figure 6 shows so-called (q, $E$ ) diagrams, which combine information about scattering intensity as a function of both energy and scattering angle (momentum transfer). Moreover, Fig. 6 also provides spatially resolved information, because Figs. 6(a) and 6(c) come from the defect-free region and Figs. 6(b) and 6(d) originate from the vicinity of the defect plane. Both the $3 \mathrm{mrad}$ collection semiangle and the $3 \mathrm{mrad}$ convergence semiangle contribute to a blurring of the phonon band-structure information contained in these images. Nevertheless, the information is still present and one can recognize dominant bands in the image. It is instructive to point out some asymmetries in the intensity of the phonon bands; for example, in Fig. 6(a) around $\Gamma$ at $90 \mathrm{mrad}$ the V-shaped band due to acoustic modes is more intense towards higher scattering angles than it is at lower scattering angles. Also, the inverted U-shaped band corresponding to optical phonons is almost not present around $\Gamma$ at $67 \mathrm{mrad}$, while it is rather pronounced near $90 \mathrm{mrad}$. Similar features have been observed in works by Senga et al. [19] and by Plotkin-Swing et al. [38]. Note, in particular, the difference in spectra around M points, when reached along the two distinct paths. Such difference is well expected, when one considers polarization vectors of the particular phonon branches (longitudinal or transversal) and their relative orientations to the propagation vector $\mathbf{q}$. Namely, the phonon scattering cross section is proportional to a scalar product of $\mathbf{q}$ and polarization vector $\boldsymbol{\varepsilon}[19,53,54]$. This is even more clearly seen for a plane-wave beam and pointlike detection (see Ref. [37]).

Differences between the defect region [Figs. 6(b) and 6(d)] and the defect-free region [Figs. 6(a) and 6(c)] seem relatively subtle. All the dominant features look very similar to each other, for both paths through the diffraction plane. A more detailed inspection reveals, however, differences near the Brillouin zone boundaries, i.e., in these plots near special points $\mathrm{K}$ and $\mathrm{M}$, respectively. Note, for example, how the noncrossing of the bands near the $\mathrm{M}$ point at $101 \mathrm{mrad}$ gets blurred in the vicinity of the defect, Fig. 6(b). Also, at low frequencies, the minima around $\mathrm{M}$ points and nearby $\mathrm{K}$ points, respectively, are visibly deeper in the defect-free region, Figs. 6(a) and 6(c), when compared to the defect region counterparts, Figs. 6(b)
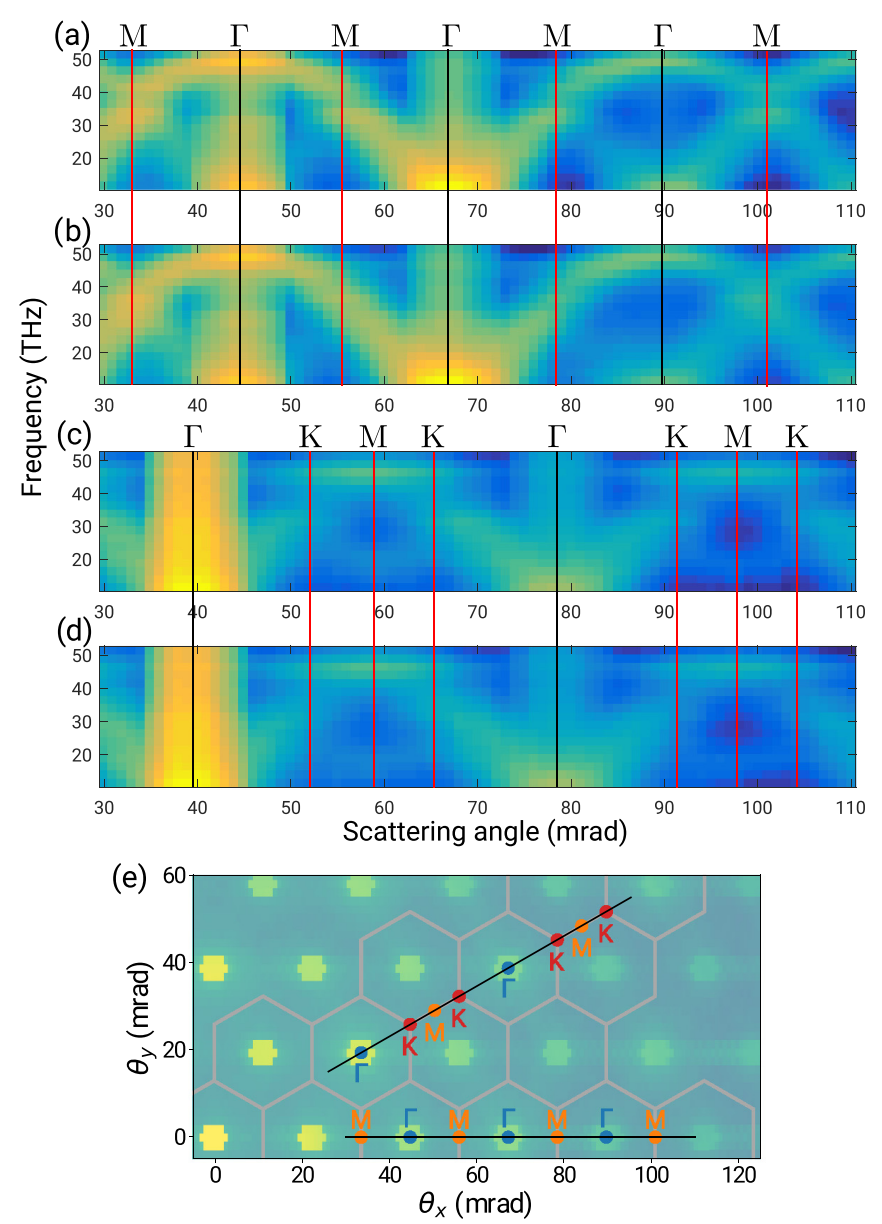

FIG. 6. (q,$E$ ) diagrams showing an evolution of local spectroscopic information as a function of scattering angle along selected directions in the $\mathbf{q}$ space: (a), (b) a scan along the $\theta_{x}$ scattering direction and (c), (d) a scan inclined under $30^{\circ}$ angle with respect to the $\theta_{x}$ direction. In each pair, the upper panel is calculated within the defect-free region and the lower one originates from the vicinity of the defect plane. The reciprocal space path of pure h-BN is mapped on the scattering directions of h-BN with defect and the vertical red lines mark the position of special points in the Brillouin zones. Subtle differences can be seen near M and K points and by generally higher intensity at lower energies within the defect region. (e) The k-space paths visualized in (a)-(d) on top of a section of a diffraction pattern calculated by the standard frozen phonon method for the nanobeam with a convergence semiangle of $3 \mathrm{mrad}$.

and 6(d). This well correlates with results shown in Fig. 5 and qualitatively with results of Ref. [24].

These differences, along with other effects, get highlighted in a one-to-one comparison. Figure 7 shows selected spectra from Fig. 6, comparing the defect-free region (red) with the defect region (blue). Several interesting observations can be made.

In Fig. 7(a) there are spectra from close neighborhoods of three different M points [see Figs. 6(a) and 6(b) at 55, 78, and $101 \mathrm{mrad}$, respectively]. First, it is striking how mutually different these three groups of spectra are. This is likely caused by dynamical diffraction, which excites individual Bragg discs with different strengths at a thickness of $15 \mathrm{~nm}$. 


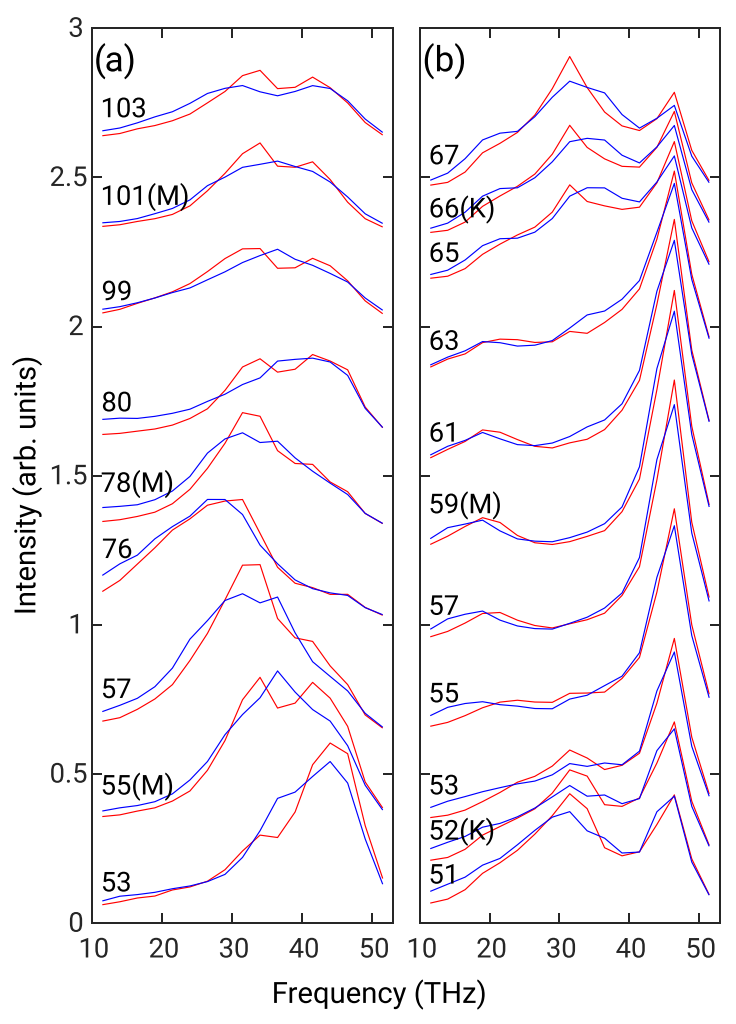

FIG. 7. Vibrational EELS spectra calculated for selected scattering angles, averaged over ten different $y$ coordinates of the beam position. Convergence and collection semiangles are both set to $3 \mathrm{mrad}$. Red lines correspond to the defect-free region and blue lines to the defect region, respectively. (a) Spectra along the horizontal path in the diffraction plane, as in Figs. 6(a) and 6(b). (b) Spectra along a path inclined $30^{\circ}$ with respect to the horizontal axis, as in Figs. 6(c) and 6(d). Scattering angles in mrad along these paths, as well as the special points, are labeled.

Without this elastic scattering effect, the intensity of inelastic scattering would decrease with increasing scattering angle due to the $1 / q^{2}$ factor in the scattering cross section. However, more intense Bragg spots may enhance inelastic scattering in their neighborhood by bringing the low- $q$ inelastic transitions to larger scattering angles. This is best seen in Fig. 6 around the (300) spot (scattering angle $\approx 66 \mathrm{mrad}$ ), where optical modes are relatively weak, while the acoustic modes in its neighborhood are stronger than around the (200) spot (scattering angle $\approx 44 \mathrm{mrad}$ ).

The second observation, which we would like to highlight here, is an apparent disappearance of the gap between the longitudinal acoustic (LA) and longitudinal optical (LO) modes between around 34 and $39 \mathrm{THz}$ (cf. Figs. 2 and 5). Note how the spectra from the defect-free region (red) consist of two peaks approximately around 34 and $41 \mathrm{THz}$, corresponding to LA and LO modes, respectively. This double-peak feature is missing in the spectra from the defect region (blue). Instead, the spectra from the defect region have usually a higher intensity at around $37 \mathrm{THz}$ than their defect-free-region counterparts, in some cases even forming a clear peak there.

In Fig. 7(b) we highlight one of the K-M-K segments from the q-space path shown Figs. 6(c) and 6(d). First, we will comment on the qualitative difference of the spectrum at the $\mathrm{M}$

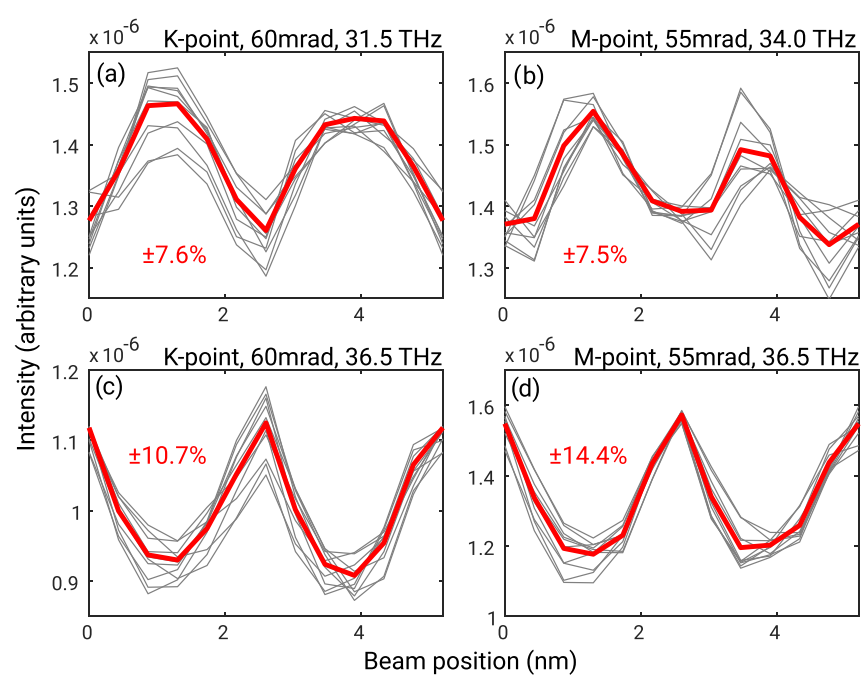

FIG. 8. Profiles of the vibrational EELS intensity across the structure model with planar defects at selected special points in the reciprocal space at specific values of energy losses. (a, c) A K-point located $66 \mathrm{mrad}$ from the direct beam along the line $30^{\circ}$ tilted from the $k_{x}$ axis [see Figs. 6(c) and 6(d)]. (b, d) An M point located $55 \mathrm{mrad}$ from the direct beam along the $k_{x}$ axis [see Figs. 6(a) and $6(\mathrm{~b})$ ]. Energy loss is indicated by the frequency bin in the top right corner of every panel. Gray lines show ten individual profiles for varying $y$ coordinates of the scan and the thick red line shows an averaged profile. Percentages refer to intensity spread of the averaged profiles, as defined in Fig. 4.

point from those in Fig. 7(a). While we see only longitudinal modes at M points in Fig. 7(a), in Fig. 7(b) we instead see the transversal modes only. This can be qualitatively understood in terms of the different relative orientation of the $\mathbf{q}$-vector and the corresponding polarization vectors $\boldsymbol{\varepsilon}$ (see a discussion and phonon band structure, Fig. 1, in Ref. [37]). In Fig. 7(b) the transversal optical (TO) mode leads to a sharp peak in the 46.5-THz energy bin and the transversal acoustic (TA) mode shows up as a weaker peak around $20 \mathrm{THz}$.

The second observation is the difference of spectral intensities around the $\mathrm{K}$ points, when comparing the spectra from the defect-free region and the defect region, especially in the energy range 30-40 THz. This correlates well with the similar qualitative feature in Fig. 5(a) calculated for a large off-axis detector; however, the flip of intensities seems to be enhanced in the surroundings of the $\mathrm{K}$ points.

We conclude this section by discussing profiles of the spectra at selected scattering angles and energy losses, where we identified in Fig. 7 notable differences between the defect and defect-free regions, respectively. Figure 8 show profiles for selected $\mathrm{K}$ and $\mathrm{M}$ points across the whole structure model. Ten profiles from individual scan lines (differing by $y$ coordinate) are shown in gray and their average is shown in red. Here we observe a much larger spread of values, when compared to the spectra calculated for a large off-axis detector in Fig. 5. This is well expected due to a significantly smaller detector area ( 3 vs $25 \mathrm{mrad}$ ), while the detector centers reside at similar scattering angles (55 and $60 \mathrm{mrad}$, respectively). By averaging over a larger number of structure snapshots the spread would likely decrease. Nevertheless, the averaged profiles (red) show 


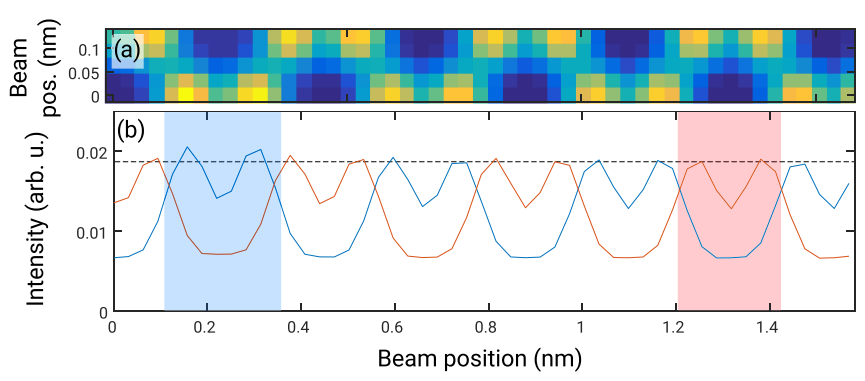

FIG. 9. (a) High-angle annular dark field image within the shaded area of the structure model, Fig. 1. (b) Line profiles from the HAADF image passing through the bottom (blue) and top (red) rows of the atomic columns. Horizontal dashed line traces intensity peaks at the positions of atomic columns in defect-free region. Only the atomic columns directly involved in the defect plane deviate from this profile. Shaded areas are used to extract averaged spectra below.

clear structure revealing the position of the defect planes. The intensity variation in these profiles is much larger than in Fig. 5, reaching about $\pm 14 \%$. That leads to a proposal that experiments with nanobeam aiming to detect an antiphase boundary in h-BN would be most likely to succeed when measuring spectra at $\mathrm{M}$ points along the horizontal path in the diffraction plane and at $\mathrm{K}$ points along the $30^{\circ}$-inclined path. We note that at higher energy resolution, both in theory as well as in experiment, the spectral differences are likely to be even more pronounced than presented here.

\section{Vibrational EELS at atomic scale}

We proceed with simulations involving an atomic-sized electron beam with convergence semiangle of $25 \mathrm{mrad}$. Neglecting any aberrations, at $60 \mathrm{kV}$ acceleration voltage, this results in a probe size of approximately $1.1 \AA$. In Fig. 1 we have highlighted an area of size approximately $1.57 \mathrm{~nm} \times$ $0.125 \mathrm{~nm}$ (green shaded and magnified region), which was scanned by a grid of $51 \times 5$ beam positions with a grid step of approximately $0.31 \AA$ in both $x$ and $y$ directions.

The advantage of using atomic-sized beams in vibrational EELS is the strong atomic-scale contrast, which can be obtained [21]. However, due to overlapping Bragg discs, it is not possible to map certain momentum transfers to certain phonon modes, since the atomic-sized convergent electron beam intermixes the vibrational signals in the diffraction plane. On the other hand, the nanometer-sized beams discussed above offer the possibility to map out phonon modes in the diffraction plane, although at a reduced spatial resolution $[18,19]$.

Analogous to the previous section, we start our analysis with an HAADF image, assuming inner and outer collection semiangles of 120 and $300 \mathrm{mrad}$, respectively (see Fig. 9). In Fig. 9(a) we show a common atomic-resolution STEMHAADF image. The atomic columns are clearly resolved. A careful eye might notice a slightly higher intensity of the two left-most atomic columns in the bottom row. Those are the columns directly involved in the APB, forming B-B and N-N bonds. This enhancement of intensity is more clearly seen in linear profiles shown in Fig. 9(b). The blue and red profiles correspond to the bottom and top lines of the HAADF image, respectively. The dotted line, which serves as a guide for the eye, shows that the intensity of almost all atomic columns is remarkably similar. Exceptions are the atomic columns directly involved in the B-B and N-N bonds, which are approximately $10 \%$ more intense, and their nearest neighbors in the upper line of the HAADF image, which are about $3 \%$ more intense. All the other atomic columns have the same intensity within the errors of the sampling (both the number of snapshots and the step size of the grid of beam positions).

These findings from Fig. 9 suggest that the defect is very localized, notably influencing only those atomic columns that are directly involved in the defect plane. The intensity change is stronger than what we have seen in Fig. 4 with a nanometer-sized beam. This could be expected due to an order of magnitude of difference in electron beam diameters. A nanometer-sized electron beam centered on the defect plane hits also atomic columns several angstroms away from the defect plane, partly blurring the local information. Thus, we would expect to see also stronger spectral changes between the defect region and the defect-free region. For that purpose we have highlighted two areas in Fig. 9(b), from which we extract averaged spectra below.

Note that Yan et al. [24] (in their extended data Fig. 1) also observe a similar intensity enhancement at the atomic scale, although their raw line profile shows a qualitatively different behavior - a locally reduced intensity oscillation with a positive offset in the vicinity of the stacking fault. We instead observe the same baseline intensity in between atomic columns and locally increased intensity in the HAADF image. This could be explained by a lower degree of alignment of atoms within atomic columns parallel to the beam direction in the vicinity of the observed SiC stacking fault, likely caused by a gradual relaxation of strain introduced by lattice mismatch of Si substrate and $\mathrm{SiC}$ grown on it.

Before proceeding to the discussion of the spectra, we note again that every atomic column in our structure model consists of the same number of $\mathrm{B}$ and $\mathrm{N}$ atoms, whether it is directly within the defect planes, or near or far from them. Seeing thus $10 \%$ differences in the intensity among these columns goes against the interpretation of the atomic-resolution HAADF images as being purely a function of composition of the columns. Note that this effect would likely not be seen within an Einstein model treatment of atomic vibrations, because it is arguably the local difference of the atomic vibration modes (an overall redshift; see Fig. 2) due to the presence of the defect. In that sense, the scenario presented here differs from the stacking fault in Ref. [24], where an atomic-resolution HAADF image clearly reveals displaced atomic columns. Here there is no such information available in the HAADF image alone and the locally increased intensity around the APB, without performing vibrational EELS, could be easily misinterpreted as a locally thicker sample. This finding may have consequences for methods related to atom counting [55].

For each beam position, at which we have evaluated the HAADF intensity, we have also calculated a full $\left(q_{x}, q_{y}, E\right)$ data cube of vibrational spectra. Figure 10 shows vibrational EELS averaged from the shaded regions within Fig. 9(b), evaluated for a large off-axis detector, similar to the experimental geometry of Hage et al. [25], specifically, a 25 mrad collection semiangle displaced by $60 \mathrm{mrad}$ along the $\theta_{x}$ direction. These spectra remind of Fig. 5 calculated with a nanometer-sized 


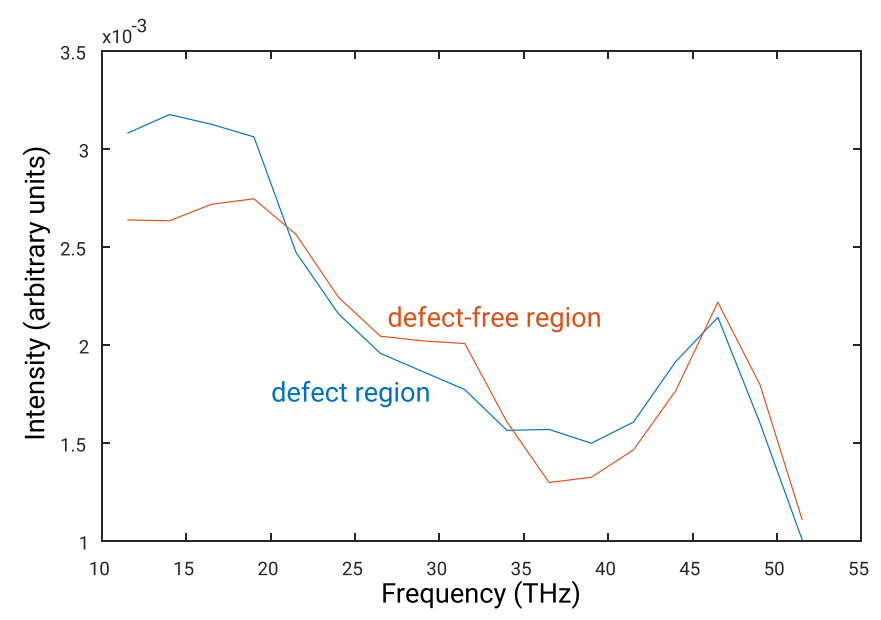

FIG. 10. Averaged spectra evaluated within the shaded regions in Fig. 9. Blue and red spectra correspond to defect and defect-free regions, respectively.

electron beam; nevertheless, as anticipated, the spectral shape differences are more pronounced here. Within the HAADF image we have seen that only the atomic columns directly involved in the defect plane show intensity differences. We can inspect whether this is the case also for vibrational EELS by plotting individual spectra along the scan lines, for which we have plotted the HAADF linear profiles in Fig. 9(b).

Figure 11 shows two-dimensional data sets, where the vertical axis represents the spectral dimension (frequency or energy loss) and the horizontal axis is the real-space coordinate. It corresponds to a line scan, where the electron beam follows the bottom scan line in Fig. 1. This type of image can be understood as a real-space analog of the $(\mathbf{q}, E)$ diagrams shown in Fig. 6. Two ways of visualizing the same data will aid the discussion below. First, we show in the top panel non-normalized raw spectra and in the bottom panel we show spectra normalized to the optical phonon peak in the 46.5-THz energy bin.

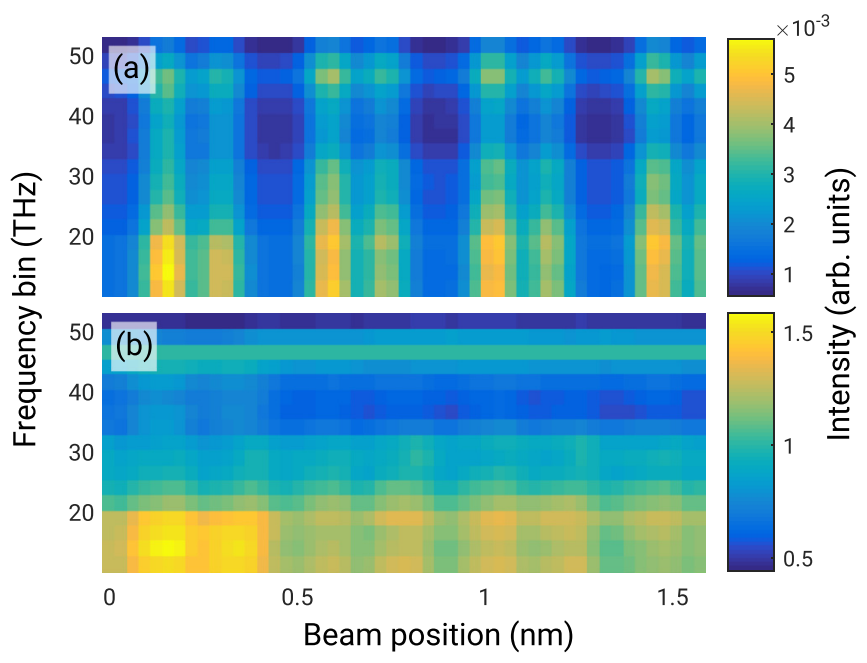

FIG. 11. (R, E) diagrams combining spectral information (vertical axis) and spatial dimension (horizontal axis). The horizontal axis corresponds to the bottom scan line from Fig. 1. Raw spectra are shown in (a), while in (b) the same spectra are shown, just normalized to the same intensity at the optical peak $(46.5 \mathrm{THz})$.
Non-normalized spectra, Fig. 11(a), show a clear atomicscale contrast with maxima at the positions of atomic columns. This agrees with experiments and calculations in Ref. [21] and with simulations in our previous work [36]. We have verified that spectra calculated at beam positions about $12 \AA$ away from the APB plane closely follow spectra obtained from an APB-free model. Focusing on the spectra from the closest vicinity of atomic columns, there is a certain enhancement of the intensity of phonon peaks below $\sim 20 \mathrm{THz}$ when the beam passes through the APB plane and its closest vicinity. We will return to this enhancement in the next paragraph. Interestingly, the pairs of neighboring atomic columns have different intensity, the left one always appearing somewhat more intense than the right one. This is caused by an asymmetric placement of the detector aperture with respect to the rest of the beam-specimen system. We have verified that centering the off-axis detector around $(-60,0) \mathrm{mrad}$ would lead to a swapping of column intensities within these pairs, making the right one more intense. The same behavior is actually also observed for pure h-BN. One can observe some additional differences between the spectra from the APB region as compared to the defect-free region; nevertheless, they are relatively subtle in this graphical representation.

The spectral signatures of the defect become more evident when we normalize all the spectra to the optical peak at 46.5 $\mathrm{THz}$, as is shown in Fig. 11(b). The spectral shape is distinctly different within the region of approximately $\pm 2 \AA$ from the center of the APB plane. Apart from the above-mentioned enhancement below $20 \mathrm{THz}$, note also the enhancement in frequency bins between 34 and $44 \mathrm{THz}$. Beyond $2 \AA$ from the center of the defect plane this local modification disappears and one can observe only slight spectral shape modifications correlated with on-column vs off-column beam positions. For instance, the relative intensity of the acoustic region with respect to the optical phonon peak is lower for beam positions in between the atomic columns.

Figure 12 presents $(\mathbf{R}, E)$ diagrams for the beam positions along the upper line scan highlighted in Fig. 1. Qualitatively the findings are similar as in Fig. 11: there is (1) the same asymmetry in the intensity of nearest-neighbor pairs of atomic columns, (2) atomic-scale spectral shape variation of the same kind, and (3) enhancement of intensity within the acoustic region for beam positions within the hexagon containing the defect plane and, in comparison to Fig. 11, a somewhat weaker enhancement of intensity within the frequency interval 34-44 THz, suggesting that these modes could be highly localized on the atoms forming B-B and N-N bonds at the defect. Note, however, that the extent of the modification of the vibrational EELS is wider than in the case of the HAADF signal (Fig. 9). There the intensity changes were almost entirely limited to the atomic columns forming the B-B and $\mathrm{N}-\mathrm{N}$ bonds, while in the case of the vibrational EELS, all atomic columns within the hexagons containing the defect plane lead to visibly modified spectra. Nevertheless, these spectral shape modifications due to the presence of the defect are still confined within a $\pm 2 \AA$ interval around the defect plane, consistent with plane-resolved LPDOS shown in Fig. 3.

The observed behavior qualitatively matches findings of Hage et al. [25] in terms of subnanometer-scale modifications 


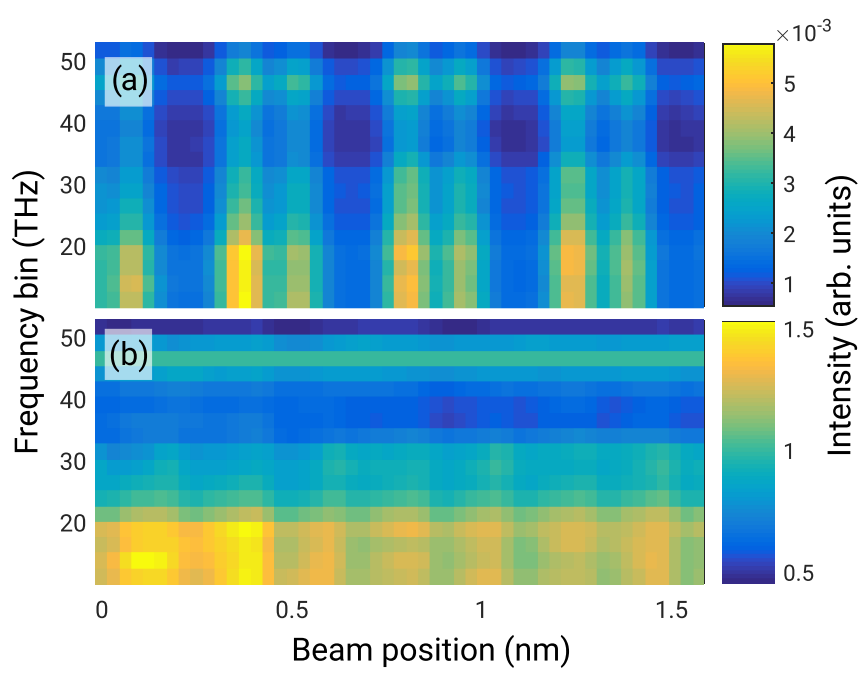

FIG. 12. ( $\mathbf{R}, E)$ diagrams along the upper scan line within the scanned area (see Fig. 1). Raw spectra are shown in (a), while in (b) the same spectra are shown, just normalized to the same intensity at the optical peak $(46.5 \mathrm{THz})$.

of the vibrational spectrum in the vicinity of a localized defect and extends it with an observation of minor atomic-scale spectral shape variations, which are likely below the sensitivity of the reported experiment.

Atomic-scale spectral shape variations were, however, observed by Venkatraman et al. [22]. They have measured vibrational EELS spectra with an on-axis detector and observed substantial atomic-scale variations in vibrational EELS for an on-axis detector geometry and a collection semiangle of $12 \mathrm{mrad}$. For a larger collection semiangle of $24 \mathrm{mrad}$, however, the spectral shape appeared to be independent of the beam position within the experimental resolution and only variations in intensity were observed.

In its present form, our method does not include dipole scattering, and therefore calculations for a polar material and for an on-axis geometry would not be realistic. Also, the on-axis geometry includes the small scattering angles, where the coherent component of the scattering cross section dominates (see our discussion related to Fig. 6 and its range of scattering angles). Nevertheless, we can probe in Fig. 13 and Figs. 14(d)-14(f) whether a reduced collection semiangle would enhance atomic-scale variations in the impact scattering regime of the vibrational EELS. These results should transfer qualitatively to nonpolar materials, such as the $\mathrm{Si}$ crystal studied by Venkatraman et al. [22]. From another perspective, we point out that the dipole scattering plays a much smaller role for large off-axis detectors as shown in Ref. [34].

In Fig. 13 we show $(\mathbf{R}, E)$ diagrams for a collection semiangle of $3 \mathrm{mrad}$ centered around a $\Gamma$ point at scattering angle $67 \mathrm{mrad}$ displaced from the center of the diffraction pattern along the $\theta_{x}$ axis. In Fig. 13(a) showing non-normalized spectra we note that in contrast with the large-collection-angle case, the intensities at atomic columns within the defect region are reduced here. Nevertheless, in the context of the previous paragraphs, it is very interesting to analyze Fig. 13(b), where all spectra are normalized to the optical peak at 46.5 THz. We observe striking differences

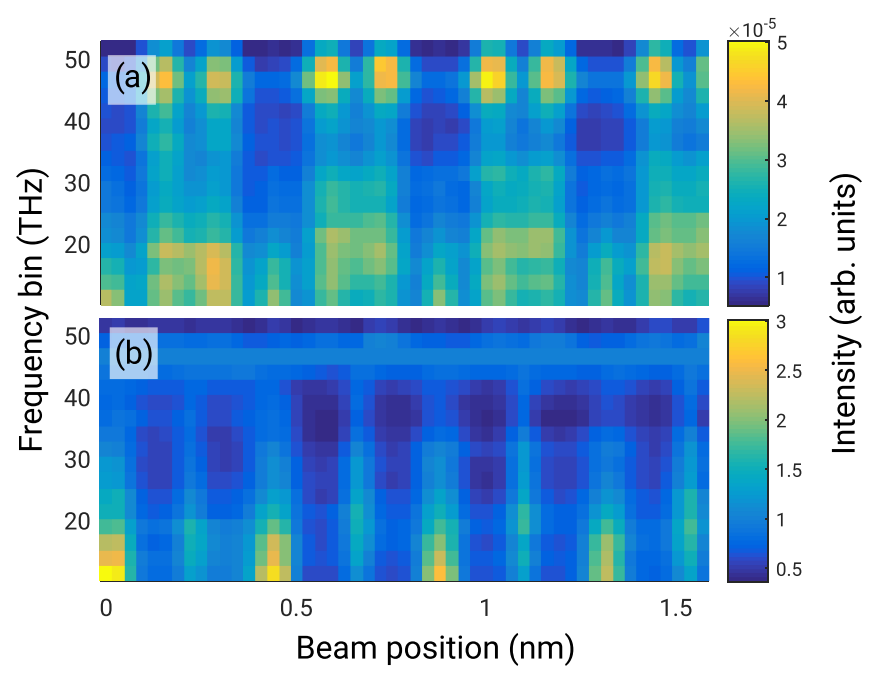

FIG. 13. ( $\mathbf{R}, E$ ) diagrams like in Fig. 11, evaluated for a $3 \mathrm{mrad}$ collection semiangle centered around the $\Gamma$ point at $\theta_{x}=67 \mathrm{mrad}$. Note the discussion around the lack of dipole scattering in these simulations in the main text for correct interpretation.

in spectral shape along the scan line. There is a notable enhancement of relative intensity at lower frequencies whenever the electron beam is in between the atomic columns. In contrast, at atomic columns the optical peak is the dominant feature. In the defect region we observe an enhancement of the relative intensity between 34 and $44 \mathrm{THz}$, especially at the position of atomic columns. Note also the range of the associated color bar, covering double the range seen in Figs. 11 and 12. Our findings thus corroborate the significant atomic-scale spectral shape variations at reduced collection semiangles reported by Venkatraman et al. [22].

Both nanometer-scale and atomic-scale spectral shape changes get highlighted in images from STEM-EELS simulations, focusing on intensities (or relative intensities) within specific frequency ranges. Figure 14 shows a collection of such STEM images for four different detector settings within three different energy ranges. For each of the four detector settings we show a STEM image formed from the intensity of the optical phonon peak at $46.5 \mathrm{THz}$ [see Figs. 14(a), 14(d), 14(g), and 14(j)]. All of them display increased intensities near the positions of atomic columns (marked with red circles) and along the nearest-neighbor bonds between them. A slight reduction of intensities can be observed near the defect plane, although the difference is rather small, as could also be expected on the basis of Fig. 10. Spectral shape differences become much more elucidated in STEM images showing relative intensities, normalized to the optical peak intensityin analogy with, e.g., Fig. 11(b). Figures 14(b) and 14(c) show such normalized STEM images for two energy intervals, namely, for 11.5-19.0 THz in Fig. 14(b) and 36.5-41.5 THz in Fig. 14(c), respectively, assuming the large 25-mrad off-axis detector discussed above. These frequency intervals coincide with light blue and rose shaded regions in Fig. 5(a). We observe that the relative intensity in those two frequency ranges is enhanced in the closest vicinity of the defect plane. Simultaneously we see the more subtle atomic-scale relative intensity changes. However, one should note the color bar 
(a) off-axis detector 25mrad @(60mrad,0), optical peak

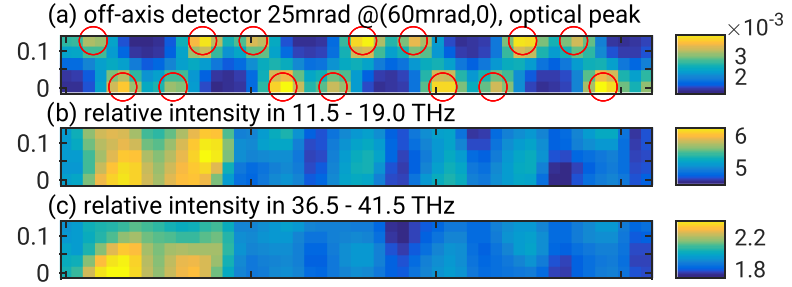

(d) 3mrad detector around $\Gamma @(67 \mathrm{mrad}, 0)$, optical peak

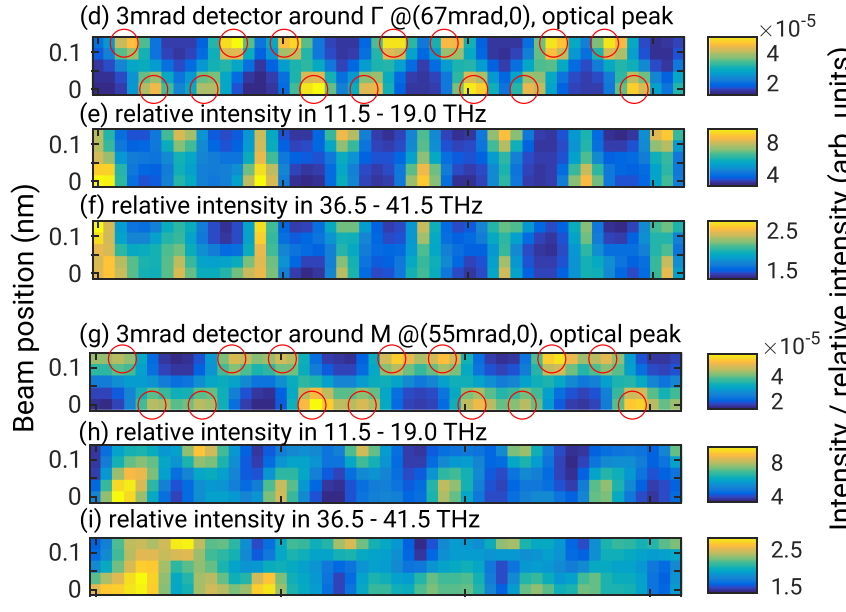

(j) 3mrad detector around K @(52,30)mrad, optical peak

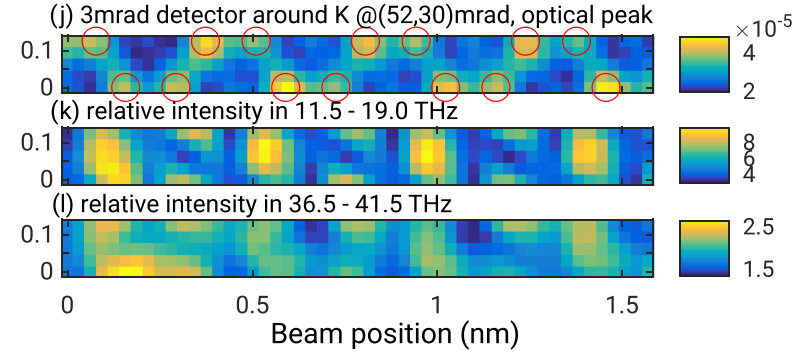

FIG. 14. Collection of STEM-EELS images for four different detector settings: (a)-(c) large off-axis detector with $25 \mathrm{mrad}$ collection semiangle centered at $(60,0) \mathrm{mrad}$, (d)-(f) detector with 3 mrad collection semiangle centered around the $\Gamma$ point at $(67,0)$ mrad, (g)-(i) the same detector centered around the M point at $(55,0)$ mrad and (j)-(1) around the $\mathrm{K}$ point at $(52,30) \mathrm{mrad}$. Within each group, the three panels show (from top to bottom) the intensity at optical peak (46.5-THz frequency bin), relative intensity within the 11.5-19.0 $\mathrm{THz}$ range normalized to the optical peak, and relative intensity within the $36.5-41.5 \mathrm{THz}$ range normalized to the optical peak. Positions of atomic columns are indicated by red circles in the panels showing optical peak intensities. Note the discussion around the lack of dipole scattering in these simulations in the main text in order to correctly interpret panels (d)-(f).

range, which spans about $20 \%$ of relative intensity variation. We have seen in Fig. 13 that relative intensity variation can be significantly larger at small collection angles.

Figures 14(d)-14(1) show STEM images for a small collection semiangle of $3 \mathrm{mrad}$, centered around selected special points in the diffraction plane, namely, the $\Gamma$ point at $(67,0)$ $\mathrm{mrad}$, the $\mathrm{M}$ point at $(55,0) \mathrm{mrad}$, and the $\mathrm{K}$ point at $(52,30)$ $\mathrm{mrad}$, respectively. The same frequency ranges have been highlighted. The optical phonon peak intensities [Figs. 14(d), $14(\mathrm{~g})$, and $14(\mathrm{j})$ ] drop by almost two orders of magnitude when compared to Fig. 14(a), in accordance with approximately 70 times lower angular coverage of the 3-mrad detector, when compared to a 25-mrad detector. Of key importance here are the relative intensity ranges, where the minimal and maximal intensity values differ by a factor of 2 or more [see Figs. 14(e) and 14(f) for the $\Gamma$ point, Figs. 14(h) and 14(i) for the M point, and Figs. 14(k) and 14(l) for the K point, respectively]. Not only do we observe significant spectral shape variations at the atomic scale, but the STEM images are also all qualitatively different. In other words, when using an electron beam of atomic size and a detector that spans a collection angle much smaller than the convergence angle and angular dimensions of the Brillouin zone, then we can observe significant spectral shape modifications within both subangstrom spatial shifts and few-millirad shifts of the detector center.

\section{CONCLUSIONS}

We have computationally analyzed a bulk hexagonal BN system with defect planes-antiphase boundaries. Distinct differences in the local phonon density of states between the in-grain and at-the-defect regions were demonstrated. These differences correlate well with the simulated vibrational electron energy loss spectra. We have shown that a nanometer-sized probe is sensitive enough to spatially resolve such spectral differences. Calculated angle-resolved spectra preserve a wealth of information about the phonon dispersions as well as their modifications due to the defect plane. These modifications are particularly pronounced at the scattering angles in the vicinity of $\mathrm{K}$ and $\mathrm{M}$ special points. Simulations with an atomic-sized electron probe offer a detailed spatial picture about the spectral shape modifications. We observe larger-scale modifications due to the presence of the defects as well as more subtle atomic-scale spectral shape variations. These variations become strongly enhanced in spectra simulated with a small collection angle.

Along with the detailed predictions of a vibrationalspectroscopic analysis of antiphase boundaries in hexagonal $\mathrm{BN}$ for large scattering angles, from a more general perspective, our results demonstrate the properties of the frequencyresolved frozen phonon multislice method-reproducing the subtleties of recent experimental works at both nanoscale and atomic resolution while being also computationally efficient, capable of dealing with a structure model containing over 22000 atoms, thanks to its linear scaling with the number of atoms.

\section{ACKNOWLEDGMENTS}

This research was financed by the Swedish Research Council through Grant Agreement No. 2017-04026. The simulations were enabled by resources provided by the Swedish National Infrastructure for Computing (SNIC), partially funded by the Swedish Research Council through Grant Agreement No. 2018-05973. 
[1] S. Ogrenci-Memik, Heat Management in Integrated Circuits: On-Chip and System-Level Monitoring and Cooling (IET Digital Library, 2015).

[2] T. N. Theis and P. M. Solomon, Science 327, 1600 (2010).

[3] M.-Y. Li, S.-K. Su, H.-S. P. Wong, and L.-J. Li, Nature (London) 567, 169 (2019).

[4] M. Maldovan, Nature (London) 503, 209 (2013).

[5] M. Haider, S. Uhlemann, E. Schwan, H. Rose, B. Kabius, and K. Urban, Nature (London) 392, 768 (1998).

[6] P. E. Batson, N. Dellby, and O. L. Krivanek, Nature (London) 418, 617 (2002).

[7] T. Akashi, Y. Takahashi, T. Tanigaki, T. Shimakura, T. Kawasaki, T. Furutsu, H. Shinada, H. Müller, M. Haider, N. Osakabe, and A. Tonomura, Appl. Phys. Lett. 106, 074101 (2015).

[8] H. Sawada, N. Shimura, F. Hosokawa, N. Shibata, and Y. Ikuhara, Microscopy 64, 213 (2015).

[9] S. Morishita, M. Mukai, K. Suenaga, and H. Sawada, Appl. Phys. Lett. 108, 013107 (2016).

[10] S. Morishita, R. Ishikawa, Y. Kohno, H. Sawada, N. Shibata, and Y. Ikuhara, Microsc. Microanal. 24, 120 (2018).

[11] O. L. Krivanek, T. C. Lovejoy, N. Dellby, T. Aoki, R. W. Carpenter, P. Rez, E. Soignard, J. Zhu, P. E. Batson, M. J. Lagos, R. F. Egerton, and P. A. Crozier, Nature (London) 514, 209 (2014).

[12] O. L. Krivanek, N. Dellby, J. A. Hachtel, J.-C. Idrobo, M. T. Hotz, B. Plotkin-Swing, N. J. Bacon, A. L. Bleloch, G. J. Corbin, M. V. Hoffman, C. E. Meyer, and T. C. Lovejoy, Ultramicroscopy 203, 60 (2019).

[13] M. J. Lagos, A. Trügler, U. Hohenester, and P. E. Batson, Nature (London) 543, 529 (2017).

[14] A. A. Govyadinov, A. Konečná, A. Chuvilin, S. Vélez, I. Dolado, A. Y. Nikitin, S. Lopatin, F. Casanova, L. E. Hueso, J. Aizpurua, and R. Hillenbrand, Nat. Commun. 8, 95 (2017).

[15] J. C. Idrobo, A. R. Lupini, T. Feng, R. R. Unocic, F. S. Walden, D. S. Gardiner, T. C. Lovejoy, N. Dellby, S. T. Pantelides, and O. L. Krivanek, Phys. Rev. Lett. 120, 095901 (2018).

[16] M. J. Lagos and P. E. Batson, Nano Lett. 18, 4556 (2018).

[17] J. A. Hachtel, J. Huang, I. Popovs, S. Jansone-Popova, J. K. Keum, J. Jakowski, T. C. Lovejoy, N. Dellby, O. L. Krivanek, and J. C. Idrobo, Science 363, 525 (2019).

[18] F. S. Hage, R. J. Nicholls, J. R. Yates, D. G. McCulloch, T. C. Lovejoy, N. Dellby, O. L. Krivanek, K. Refson, and Q. M. Ramasse, Sci. Adv. 4, 6 (2018).

[19] R. Senga, K. Suenaga, P. Barone, S. Morishita, F. Mauri, and T. Pichler, Nature (London) 573, 247 (2019).

[20] K. Venkatraman and P. A. Crozier, Microsc. Microanal. 1 (2021).

[21] F. S. Hage, D. M. Kepaptsoglou, Q. M. Ramasse, and L. J. Allen, Phys. Rev. Lett. 122, 016103 (2019).

[22] K. Venkatraman, B. D. A. Levin, K. March, P. Rez, and P. A. Crozier, Nat. Phys. 15, 1237 (2019).

[23] S. M. Collins, D. M. Kepaptsoglou, J. Hou, C. W. Ashling, G. Radtke, T. D. Bennett, P. A. Midgley, and Q. M. Ramasse, Nano Lett. 20, 1272 (2020).
[24] X. Yan, C. Liu, C. A. Gadre, L. Gu, T. Aoki, T. C. Lovejoy, N. Dellby, O. L. Krivanek, D. G. Schlom, R. Wu, and X. Pan, Nature (London) 589, 65 (2021).

[25] F. S. Hage, G. Radtke, D. M. Kepaptsoglou, M. Lazzeri, and Q. M. Ramasse, Science 367, 1124 (2020).

[26] P. Rez, Ultramicroscopy 52, 260 (1993).

[27] P. Rez, Microsc. Microanal. 20, 671 (2014).

[28] P. Rez and A. Singh, Ultramicroscopy 220, 113162 (2021).

[29] B. D. Forbes, A. V. Martin, S. D. Findlay, A. J. D'Alfonso, and L. J. Allen, Phys. Rev. B 82, 104103 (2010).

[30] N. R. Lugg, B. D. Forbes, S. D. Findlay, and L. J. Allen, Phys. Rev. B 91, 144108 (2015).

[31] B. D. Forbes and L. J. Allen, Phys. Rev. B 94, 014110 (2016).

[32] C. Dwyer, Phys. Rev. B 89, 054103 (2014).

[33] C. Dwyer, T. Aoki, P. Rez, S. L. Y. Chang, T. C. Lovejoy, and O. L. Krivanek, Phys. Rev. Lett. 117, 256101 (2016).

[34] C. Dwyer, Phys. Rev. B 96, 224102 (2017).

[35] A. Konečná, F. Iyikanat, and F. J. García de Abajo, ACS Nano 15, 9890 (2021).

[36] P. M. Zeiger and J. Rusz, Phys. Rev. Lett. 124, 025501 (2020).

[37] P. M. Zeiger and J. Rusz, Phys. Rev. B 104, 104301 (2021).

[38] B. Plotkin-Swing, G. J. Corbin, S. De Carlo, N. Dellby, C. Hoermann, M. V. Hoffman, T. C. Lovejoy, C. E. Meyer, A. Mittelberger, R. Pantelic, L. Piazza, and O. L. Krivanek, Ultramicroscopy 217, 113067 (2020).

[39] Y. Liu, X. Zou, and B. I. Yakobson, ACS Nano 6, 7053 (2012).

[40] L. C. Gomes, S. S. Alexandre, H. Chacham, and R. W. Nunes, J. Phys. Chem. C 117, 11770 (2013).

[41] LAMMPS website, http://lammps.sandia.gov.

[42] J. H. Los, J. M. H. Kroes, K. Albe, R. M. Gordillo, M. I. Katsnelson, and A. Fasolino, Phys. Rev. B 96, 184108 (2017).

[43] C. Sevik, A. Kinaci, J. B. Haskins, and T. Cagin, Phys. Rev. B 84, 085409 (2011).

[44] W. Ouyang, I. Azuri, D. Mandelli, A. Tkatchenko, L. Kronik, M. Urbakh, and O. Hod, J. Chem. Theory Comput. 16, 666 (2020).

[45] T. Maaravi, I. Leven, I. Azuri, L. Kronik, and O. Hod, J. Phys. Chem. C 121, 22826 (2017).

[46] PHONOLAMMPS github repository, https://github.com/ abelcarreras/phonolammps, version 0.5.2.

[47] PHONOPY github repository, https://github.com/phonopy/ phonopy, version 2.4.2.

[48] A. Togo and I. Tanaka, Scr. Mater. 108, 1 (2015).

[49] R. Dettori, M. Ceriotti, J. Hunger, C. Melis, L. Colombo, and D. Donadio, J. Chem. Theory Comput. 13, 1284 (2017).

[50] GLE input generator, http://gle4md.org/index.html?page= matrix.

[51] J. Barthel, Ultramicroscopy 193, 1 (2018).

[52] R. F. Loane, P. Xu, and J. Silcox, Acta Crystallogr. Sect. A 47, 267 (1991).

[53] C. Dwyer, Microsc. Microanal. 24, 410 (2018).

[54] R. J. Nicholls, F. S. Hage, D. G. McCulloch, Q. M. Ramasse, K. Refson, and J. R. Yates, Phys. Rev. B 99, 094105 (2019).

[55] A. De Backer, G. Martinez, A. Rosenauer, and S. Van Aert, Ultramicroscopy 134, 23 (2013). 